\title{
Serotonin Syndrome Developing Immediately after the Initiation of Low-Dose Methadone Therapy: A Case Report
}

\author{
Masayoshi Kumai ${ }^{a}$ b Yosuke Maeda ${ }^{b}$ Mototsugu Miura ${ }^{b}$ \\ Kenkichi Tsurugab Takehiro Yamada ${ }^{c}$ Yoh Takekuma ${ }^{a}$ \\ Mitsuru Sugawaraa, d \\ ${ }^{a}$ Department of Pharmacy, Hokkaido University Hospital, Sapporo, Japan; b Cancer Center \\ Palliative Care Team, Hokkaido University Hospital, Sapporo, Japan; 'Department of \\ Pharmacotherapy, Faculty of Pharmaceutical Sciences, Hokkaido University of Science, \\ Sapporo, Japan; 'Laboratory of Pharmacokinetics, Faculty of Pharmaceutical Sciences, \\ Hokkaido University, Sapporo, Japan
}

\section{Keywords}

Serotonin syndrome $\cdot$ Methadone $\cdot$ Oxycodone

\begin{abstract}
We present a case in which serotonin syndrome developed immediately after the initiation of low-dose methadone following an increase in oxycodone dose and the initiation of duloxetine. The symptoms of serotonin syndrome were alleviated and later disappeared upon cessation of methadone alone. The case was a 47-year-old woman with a desmoid tumor. The administration of duloxetine ( $20 \mathrm{mg} /$ day) was initiated while the patient took oxycodone sustained-release tablets ( $40 \mathrm{mg} /$ day). The following day, excessive perspiration, chills, and tremors appeared after the initiation of $15 \mathrm{mg} /$ day methadone. Discontinuation of methadone led to an alleviation of the symptoms which completely disappeared 3 days later. The results suggest that low-dose methadone can trigger serotonin syndrome as early as after the first dose. Thus, it is important to be aware of the risks and to immediately take action if symptoms appear.

(C) 2020 The Author(s).

Published by S. Karger AG, Basel
\end{abstract}

\section{Introduction}

Serotonin syndrome (SS) is an adverse event triggered by the use of molecules such as selective serotonin reuptake inhibitors and serotonin-norepinephrine reuptake inhibitors and causes some symptoms such as excessive perspiration, clonus, and tremors due to 
excessive serotonin in the central nervous system [1]. In recent years, opioids including methadone have been reported to have an inhibitory effect on the serotonin transporter (SERT), a factor that plays an important role in the reuptake of serotonin in the nerve endings $[2,3]$. In 2016, the United States Food and Drug Administration (FDA) released a Drug Safety Communication to warn the medical community of the SS risk that is secondary to opioid use [4].

Herein, we present a case in which SS developed immediately after the initiation of low-dose methadone following an increase in oxycodone dose and initiation of duloxetine. The symptoms of SS were alleviated and later disappeared upon the cessation of methadone alone.

\section{Case Report}

The patient was a 47-year-old Japanese woman who noticed a mass in her left neck; MRI revealed a tumor that had deeply invaded beyond the deep cervical fascia. A biopsy revealed a pathological diagnosis of a desmoid tumor 10 months before this hospital admission. Five months prior to the admission, the patient was treated with anti-estrogen and nonsteroidal anti-inflammatory agents; however, the treatment was discontinued due to severe dizziness. Radiotherapy was initiated 3 months prior to this hospital admission, but the tumor showed no evidence of shrinkage. The patient was therefore admitted to the hospital for additional pharmacotherapy.

The chief complaints during this hospitalization were pain accompanied by allodynia on the left cervical skin and numbness of the left arm. The drugs administered at the time of admission included oxycodone sustained-release tablets $20 \mathrm{mg} /$ day, oxycodone immediaterelease powder (5 $\mathrm{mg}$ as needed for pain), pregabalin capsules $100 \mathrm{mg} /$ day, and esomeprazole capsules $20 \mathrm{mg} /$ day.

On hospitalization day 1 , the dose of oxycodone sustained-release tablets was increased to $40 \mathrm{mg}$ /day and celecoxib tablets $200 \mathrm{mg}$ /day were initiated due to extreme pain. On hospitalization day 2, pregabalin capsules were discontinued and duloxetine capsules $20 \mathrm{mg} /$ day (once a day in the morning after the meal) were initiated. Because nausea was observed, the administration of prochlorperazine tablets $15 \mathrm{mg}$ /day was initiated together with magnesium oxide tablets $2 \mathrm{~g}$ /day to prevent constipation. On hospitalization day 3, duloxetine administration was changed to after the evening meal from the next day due to complaints of sleepiness and lightheadedness. Furthermore, on hospitalization day 3, methadone therapy was initiated with the intent to continue oxycodone until methadone levels reached the target concentration in serum; oxycodone would then be discontinued and a course of methadone $15 \mathrm{mg} /$ day (5 mg thrice a day) would be initiated. Following the initial dose of methadone (5 $\mathrm{mg}$ ) at 13:00 $\mathrm{h}$ and a second dose at 20:00 h, chills, tremors, and excessive perspiration were observed at 22:00 h. Because these symptoms repeatedly disappeared and then returned, methadone was discontinued. On hospitalization day 4, chills and tremors disappeared but there was excessive perspiration, although it improved to some degree. Excessive perspiration continued throughout hospitalization day 5 and finally disappeared on day 6 . SS diagnosis was made as per the Sternbach diagnostic criteria [5]. Specifically, chills, tremors, and excessive perspiration appeared and disappeared in conjunction with the initiation and discontinuation of methadone therapy. In addition, the patient did not receive antipsychotic drug therapy or experience any change in the therapeutic regimen; infection, metabolic disorder, and/or withdrawal following substance abuse were ruled out. 


\section{Discussion}

Methadone has an inhibitory effect on SERT $[2,3]$. Methadone administration is categorized as an intermediate risk factor for SS according to a study by Baldo and Rose [2]. In addition, a Drug Safety Communication released by the FDA in 2016 covering cases of opioidrelated SS in the FDA Adverse Event Reporting System database from 1969 through 2013 included 5 cases secondary to methadone and 7 cases secondary to oxycodone [4].

SS resulting from the combined use of duloxetine and methadone, as in the present case, has previously been reported [6]. However, this earlier report described SS secondary to multiple years of methadone, duloxetine, and desipramine use, and the patient had a methadone overdose. There are other reports on SS involving the combined use of methadone with other drugs [7-9], but in most cases the additional causative drug was administered over a long period of time and methadone was administered at high doses. We did not find any report describing the onset of SS immediately after initiating methadone at a minimum dose, as described in the present case.

Our case involved an increase in the oxycodone dose and initiation of duloxetine followed by methadone over a brief period of time. SS is likely to occur soon after the initiation of the drug [10]; polypharmacy is also a known risk factor [11]. In the present case, we cannot say for certain that methadone alone was the inciting factor; however, SS improved and eventually disappeared after the discontinuation of methadone alone while duloxetine and oxycodone were continued at the same dose. This result suggests that methadone was an important trigger in developing SS in our patient.

The incidence of SS is low; however, if the disease becomes serious, it can be life-threatening. SS presents with a variety of symptoms, and to make a definitive diagnosis can take a considerable amount of time. Of note, the package insert of methadone in the United States and Japan does not list SS. The findings of the present case suggest that physicians and patients alike should be aware of developing a risk of SS immediately following the initiation of low-dose methadone therapy and take action immediately when symptoms appear to prevent a worsening of the disease.

\section{Statement of Ethics}

This case report was conducted in accordance with the Declaration of Helsinki. Informed consent was obtained in writing from the patient for the publication of the clinical data.

\section{Disclosure Statement}

The authors have no conflicts of interest to declare.

\section{Funding Sources}

No funding was received for this research. 
Kumai et al.: Serotonin Syndrome Immediately after Methadone Therapy

\section{Author Contributions}

Masayoshi Kumai treated the patient, designed the research study, drafted the manuscript, and contributed to the interpretation of data. Yosuke Maeda, Mototsugu Miura, and Kenkichi Tsuruga treated the patient, revised the manuscript, and contributed to the interpretation of data. Yoh Takekuma and Takehiro Yamada revised the manuscript and contributed to the interpretation of data. Mitsuru Sugawara supervised the entire project and completed the article. All authors have read and approved the final manuscript.

\section{References}

1 Isbister GK, Buckley NA, Whyte IM. Serotonin toxicity: a practical approach to diagnosis and treatment. Med J Aust. 2007 Sep;187(6):361-5.

2 Baldo BA, Rose MA. The anaesthetist, opioid analgesic drugs, and serotonin toxicity: a mechanistic and clinical review. Br J Anaesth. 2020 Jan;124(1):44-62.

3 Rickli A, Liakoni E, Hoener MC, Liechti ME. Opioid-induced inhibition of the human 5-HT and noradrenaline transporters in vitro: link to clinical reports of serotonin syndrome. Br J Pharmacol. 2018 Feb;175(3):532-43.

4 FDA Drug Safety Communication [Internet]. FDA warns about several safety issues with opioid pain medicines; requires label changes [cited 2019 Nov 10]. Available from: https://www.fda.gov/drugs/drug-safetyand-availability/fda-drug-safety-communication-fda-warns-about-several-safety-issues-opioid-pain-medicines-requires.

5 Sternbach H. The serotonin syndrome. Am J Psychiatry. 1991 Jun;148(6):705-13.

6 Rastogi R, Swarm RA, Patel TA. Case scenario: opioid association with serotonin syndrome: implications to the practitioners. Anesthesiology. 2011 Dec;115(6):1291-8.

7 Mastroianni A, Ravaglia G. Serotonin syndrome due to co-administration of linezolid and methadone. Infez Med. 2017 Sep 1;25(3):263-6.

8 Hillman AD, Witenko CJ, Sultan SM, Gala G. Serotonin syndrome caused by fentanyl and methadone in a burn injury. Pharmacotherapy. 2015 Jan;35(1):112-7.

9 Bush E, Miller C, Friedman I. A case of serotonin syndrome and mutism associated with methadone. J Palliat Med. 2006 Dec;9(6):1257-9.

10 Wang RZ, Vashistha V, Kaur S, Houchens NW. Serotonin syndrome: preventing, recognizing, and treating it. Cleve Clin J Med. 2016 Nov;83(11):810-7.

11 Lane R, Baldwin D. Selective serotonin reuptake inhibitor-induced serotonin syndrome: review. J Clin Psychopharmacol. 1997 Jun;17(3):208-21. 